can report them. Contact information should include the IACUC Chair Person, Attending Veterinarian, other institutional veterinarians, and animal care managers and supervisors. Any allegations of animal-related wrongdoing made anonymously should be kept that way as best as possible. The research institution and IACUC should have a plan in place to shield whistleblowers that in good faith come forward with concerns.
Research employee protections matter and should be established. Any individual working in these environments and witness wrongdoing should be willing to come forward without fear of retaliation. The whistleblower is the gatekeeper in the workplace and should be protected.

\section{Jeffrey Etue ${ }^{\bowtie}$}

University of Kentucky, Lexington, KY, USA.

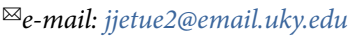

Published online: 27 May 2020

https://doi.org/10.1038/s41684-020-0554-9

References

1. Code of Federal Regulations. Title 9, Chapter 1, Subchapter a Animal Welfare: Part 2 regulations (\$2.31).

2. Public Health Service. Policy on Humane Care and Use of Laboratory Animals. (US Department of Health and Human Services, Washington, DC. 1986, amended 2002).

3. Office of Laboratory Animal Welfare. What Investigators Need to Know About the Use of Animals. https://grants.nih.gov/ grants/olaw/Investigatorsneed2Know.pdf

\title{
The problem with a murky whistleblower policy
}

W hile OLAW cannot guarantee protection from reprisals, OLAW does refer to the Guide ${ }^{1}$ (p. 24), which states that "mechanisms for reporting concerns should be posted in prominent locations in the facility and on applicable institutional website(s) with instructions on how to report the concern and to whom. Multiple points of contact, including senior management, the IO, IACUC Chair, and AV, are recommended."

Richmond did the right thing by reporting the noncompliance to the IACUC. Levine is using 'guilt by association' to punish Richmond, as only Levine and Richmond knew about the noncompliance. Richmond told the whole truth when he admitted to performing the procedure. There is nothing inherently wrong with anonymity, so admitting that he reported the noncompliance is not necessary. Richmond has seen his employer's true colors. We would encourage him to find a lab that is more ethical, as well as aware of current legal and appropriate HR practices.

As OLAW is not the employer, they would not be able to guarantee there were no reprisals at the institutional level. However, the Guide does further refine OLAW's expectations, stating “The process should include a mechanism for anonymity, compliance with applicable whistleblower policies, nondiscrimination against the concerned/reporting party, and protection from reprisals." We assume that Great Eastern University has an Assurance on file with OLAW. Because of this, they are bound by the principles in the Guide, and therefore, should have "protection from reprisals" documented in their whistleblower policy.

The IACUC needs to revisit their policy on whistleblowers to include protection from reprisals and ensure it is consistent with the rest of OLAW and the Guide's recommendations. To protect anonymous reporters, outlining examples of reprisal, such as termination of employment of lab members without adequate justification, will help avoid similar situations in the future. The investigation into non-compliance in Levine's studies should continue, but it may proceed as post approval monitoring. Considering this investigator's temperament and willingness to overlook one area of non-compliance, it is not unreasonable to think there may be other protocol non-compliances.
If the IACUC does not already have one, a written policy on how non-compliances are dealt with would be advantageous. The IACUC should also consult with their legal department to revise the university's bylaws, as they are in direct conflict with a federal mandate. On a slightly separate note, the PI needs to be trained from Human Resources on appropriate employee termination practices.

\section{Christine Boehm ${ }^{1 凶}$ and Kristina Pugh ${ }^{2}$}

${ }^{1}$ Texas Tech University Health Science Center at El Paso, El Paso, TX, USA. ${ }^{2}$ William Beaumont Army Medical Center, El Paso, TX, USA.

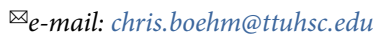

Published online: 27 May 2020

https://doi.org/10.1038/s41684-020-0552-y

References

1. Institute for Laboratory Animal Research. Guide for the Care and Use of Laboratory Animals. 8th edition (National Academies Press, Washington, D.C., USA, 2011).

\section{Disclaimer}

This information represents the views of the author. The information or content presented does not represent the official position or policy of the U.S. Army Medical Department, the Department of Defense or the U.S. Government.

\section{Coming clean}

T o maintain public trust in the welfare and ethical treatment of animals used in biomedical research, it is imperative that institutions have policies that explicitly forbid reprisal against whistleblowers for reporting noncompliance and humane concerns. In the case of Joshua Richmond, it is unfortunate that the IACUC's policy did not address reprisal, especially since the broader institution also does not seem to have any such policy. The Office of Laboratory Animal Welfare (OLAW) assigns responsibility against reprisals to the institution, and the IACUC is the delegated authority to make recommendations and develop policies specific to its areas of animal care program oversight, consistent with the Guide. If
Great Eastern University has a valid OLAW assurance on file, it would clearly state that they are in compliance with the Guide such that nondiscrimination against the concerned/reporting party and protection from reprisals is enforced ${ }^{1,2}$.

Because Richmond did not identify himself as the whistleblower, such a policy may still not have helped him, as Levine 